\title{
Probability density estimation in stochastic environmental models using reverse representations
}

Published online: 20 November 2005

(C) Springer-Verlag 2005

\begin{abstract}
The estimation of probability densities of variables described by stochastic differential equations has long been done using forward time estimators, which rely on the generation of forward in time realizations of the model. Recently, an estimator based on the combination of forward and reverse time estimators has been developed. This estimator has a higher order of convergence than the classical one. In this article, we explore the new estimator and compare the forward and forward-reverse estimators by applying them to a biochemical oxygen demand model. Finally, we show that the computational efficiency of the forward-reverse estimator is superior to the classical one, and discuss the algorithmic aspects of the estimator.
\end{abstract}

\section{Introduction}

Many environmental models used today are based on deterministic differential equations (Loucks et al.1981). However, the abilities of these models for prediction in real life applications, are often hampered by uncertainties in initial conditions, model parameters and/or sources. Decision makers have also recognized the inherent uncertainty in modeling efforts and they are more and more asking for risk estimates. For example, in a water pollution problem, they want to know the probability of exceeding a critical concentration of a substance.

E. van den. Berg · A. W. Heemink $(\bowtie) \cdot H$. X. Lin Department of Applied Mathematical Analysis,

Delft University of Technology Faculty of Information

Technology and Systems, Mekelweg 4, 2628 CD Delft,

The Netherlands

E-mail: A.W.Heemink@its.tudelft.nl

Tel.: + 31-15-2785813

Fax: + 31-15-2787295

J. G. M. Schoenmakers

Weierstrass Institute for Applied Analysis and Stochastics, Mohrenstrasse 39, 10117 Berlin, Germany
Uncertainties can be included into the model by introducing noise processes as forcing terms. As a result a stochastic differential equation is obtained (Jazwinsky 1970). In general stochastic differential equations cannot be solved analytically and have to be approximated numerically to derive a discrete stochastic model (Kloeden and Platen 1992). A well-known technique for estimating probabilistic characteristics of a discrete stochastic model is Monte Carlo simulation (Hammersley and Handscomb 1964). Here many different realizations of the stochastic model are generated to get information about the probability density of the model results. This approach is conceptually very simple and can be applied for many different types of (highly nonlinear) problems. However, Monte Carlo techniques do consume a large amount of CPU time and the accuracy of the estimates improves very slowly with the increase of sample size.

The efficiency of Monte Carlo methods can be improved by variance reduction (Kloeden and Platen 1992). Here an approximation of the Kolmogorov backwards equation is required. In some simple applications analytical approximations of this partial differential equation can be used. In general, however, a numerical solution is required. For high dimensional systems this may become very time consuming (Schoenmakers et al 2002), (Milstein and Schoenmakers 2002).

Recently, (Milstein et al. 2002) introduced the concept of reverse time diffusion. The classical Monte Carlo estimate is based on forward realizations of the original stochastic model. (Milstein et al. 2002) derived a reverse system from the original model and showed that the classical Monte Carlo estimate can also be based on realization of this reverse system. For many applications it is more efficient to use realizations of the reverse system instead of the original model. The most efficient implementation is, however, obtained if the forward realizations and the reverse system realizations are combined. This is called the forward-reverse estimator. Estimators based on reverse time realization are very attractive if the probability density is required at a very 
limited number of time steps and at a very limited number of exceedence levels.

In this paper, we apply the forward-reverse method to estimate the probability density of a stochastic biochemical-oxygen demand (BOD) model. In Sect. 2, we describe the classical Monte Carlo estimation, present a general probabilistic representation of this estimator based on forward realizations and introduce the forward-reverse estimator. In Sect. 3, the BOD model is given, and its reverse model is derived. We describe in detail an application of the method to estimate the probability density of the BOD model in Sect. 4 and compare the performance of the forward with forwardreverse estimators in Sect. 5.

\section{Transition density estimators for SDEs}

Consider the stochastic differential equation (SDE) in the Itô sense

$\mathrm{d} X=a(s, X) d s+\sigma(s, X) \mathrm{d} W(s), t_{0} \leq s \leq T, X\left(t_{0}\right)=x_{0}$

where $X=\left(X^{1}, \ldots, X^{d}\right)^{\top}, a=\left(a^{1}, \ldots, a^{d}\right)^{\top}$ are $d$-dimensional vectors, $W=\left(W^{1}, \ldots, W^{m}\right)^{\top}$ is an $m$-dimensional standard Wiener process, and $\sigma=\left\{\sigma^{i j}\right\}$ is a $d \times m$-matrix, $m \geq d$. We assume that the $d \times d$-matrix $b:=\sigma \sigma^{\top}, b=$ $\left\{b^{i j}\right\}$, is of full rank for every $(s, x), s \in\left[t_{0}, T\right], x \in R^{d}$. The functions $a^{i}(s, x)$ and $\sigma{ }^{i j}(s, x)$ are assumed to be sufficiently smooth such that we have existence and uniqueness of the solution $X_{t, x}(s) \in R^{d}, X_{t, x}(t)=x, t_{0} \leq$ $t \leq s \leq T$, of (1), smoothness of the transition density $p(t, x, s, y)$ of the Markov process $X$, and existence of all the moments of $p(\cdot, \cdot, y)$.

The solution of SDE ((1)) may be approximated by different numerical methods, see (Kloeden and Platen 1992; Milstein 1995). Here we consider the Euler scheme,

$$
\begin{aligned}
X\left(t_{k+1}\right)= & X\left(t_{k}\right)+a\left(t_{k}, X\left(t_{k}\right)\right)\left(t_{k+1}-t_{k}\right) \\
& +\sigma\left(t_{k}, X\left(t_{k}\right)\right) \sqrt{t_{k+1}-t_{k}} \varsigma_{k},
\end{aligned}
$$

with $t_{0}<t_{1}<\ldots<t_{K}=T$, and $\varsigma_{k} \in R^{m}, k=0, \ldots, K-1$, being i.i.d. standard normal random variables. In fact, (2) is the most simple numerical scheme for integration of SDE (1).

\subsection{Classical and non-classical density estimators}

We now review some known estimators for the density $p(t, x, T, y)$ in connection with (1).

\subsubsection{The Parzen-Rosenblatt (pure forward or classical) estimator}

Let $\bar{X}_{t, x}$ be a numerical approximation of $X_{t, x}$ obtained by the Euler method and let $\bar{X}_{n}:=\bar{X}_{t, x}^{(n)}(T), n=1, \ldots, N$, be a sample of independent realizations of $\bar{X}_{t, x}(T)$. Then one may estimate the transition density $p(t, x, T, y)$ from this sample by using standard techniques of non-parametric statistics such as the classical kernel (Parzen-Rosenblatt) estimator. The kernel (Parzen-Rosenblatt) density estimator with a kernel $K$ and a bandwidth $\delta$ is given by

$\hat{p}_{F E}(t, x, T, y)=\frac{1}{N \delta^{d}} \sum_{n=1}^{N} K\left(\frac{\bar{X}_{n}-y}{\delta}\right)$,

see (Devroye and Györfi 1985), (Silverman 1986). For example, in (3) one could take the Gaussian kernel $K(x)=(2 \pi)^{-d / 2} \exp \left(-|x|^{2} / 2\right)$. Here $\delta$ should decrease to zero as $N$ increases while $N \delta^{d} \rightarrow \infty$, see (Devroye 1986).

\subsubsection{The pure reverse estimator ( $R E$ )}

In order to proceed with more sophisticated density estimators we introduce a reverse diffusion system for (1). In a more special setting the notion of reverse or adjoint diffusion is due to Thomson (Thomson 1987). The reverse system introduced in this paper can be seen as a generalization of Thomson's approach and is derived in (Milstein, Schoenmakers and Spokoiny 2002) in a more transparent and more rigorous way. We first introduce a reversed time variable $\tilde{s}=T+t-s$ and define the functions

$\tilde{a}^{i}(\tilde{s}, y)=a^{i}(T+t-\tilde{s}, y)$,

$\tilde{b}^{i j}(\tilde{s}, y)=b^{i j}(T+t-\tilde{s}, y)$.

For a vector process $Y_{t, y}(s) \in I R^{d}$ and a scalar process $\mathscr{Y}_{t, y}(s)$ we then consider the reverse time stochastic system

$$
\begin{array}{cc}
\mathrm{d} Y=\alpha(s, Y) \mathrm{d} s+\tilde{\alpha}(s, Y) \mathrm{d} \tilde{W}(s), & Y(t)=y, \\
\mathrm{~d} \mathscr{Y}=c(s, Y) \mathscr{Y} \mathrm{d} s, & \mathscr{Y}(t)=1,
\end{array}
$$

with $\tilde{W}$ being an $m$-dimensional standard Wiener process and

$\alpha^{i}(s, y)=\sum_{j=1}^{d} \frac{\partial \tilde{b}^{i j}}{\partial y^{j}}-\tilde{a}^{i}$,

$c(s, y)=\frac{1}{2} \sum_{i, j=1}^{d} \frac{\partial^{2} \tilde{b}^{i j}}{\partial y^{i} \partial y^{j}}-\sum_{i=1}^{d} \frac{\partial \tilde{a}^{i}}{\partial y^{i}}$,

$\tilde{\sigma}(s, y)=\sigma(T+t-s, y)$

It is possible to construct an alternative density estimator in terms of the reverse system (4). Suppose that $\left(\bar{Y}_{0, y}^{(m)}, \overline{\mathscr{Y}}_{0, y}^{(m)}\right), m=1, \ldots, M$, is an i.i.d. sample of numerical solutions of (4) starting at $t=0$, obtained by the Euler scheme. Then a pure reverse estimator is given by

$\hat{p}_{R E}(t, x, T, y):=\frac{1}{M \delta^{d}} \sum_{m=1}^{M} K\left(\frac{x-\bar{Y}_{0, y}^{(m)}(T)}{\delta}\right) \overline{\mathscr{Y}}_{0, y}^{(m)}(T)$. 
In fact, the reverse estimator (8) can be obtained as a side case from the forward-reverse estimator discussed discussed in $\mathrm{C}$.

\subsubsection{The forward-reverse estimator (FRE)}

By combining the forward (1) and reverse (4) estimators via the Chapman-Kolmogorov equation with respect to an intermediate time $t^{*}$, (Milstein, Schoenmakers, and Spokoiny 2002) constructed the so called FRE,

$$
\begin{gathered}
\hat{p}_{F R E}(t, x, T, y)=\frac{1}{M}\left[\frac{1}{N \delta^{d}} \sum_{m=1}^{M} \sum_{n=1}^{N} K\left(\frac{\bar{X}_{t, x}^{(n)}\left(t^{*}\right)-\bar{Y}_{t^{*}, y}^{(m)}(T)}{\delta}\right)\right. \\
\left.\times \overline{\mathscr{Y}}_{t^{*}, y}^{(m)}(T)\right] .
\end{gathered}
$$

It is shown that the FRE (9) has superior properties in comparison with density estimators based on pure forward (3) or pure reverse (8) representations. Obviously, by taking $t^{*}=T$ and $t^{*}=0$, the estimator (9) collapses to the pure forward estimator (3) and pure reverse estimator (8), respectively.

\subsection{Theoretical properties of the estimators}

\subsubsection{Order of convergence.}

In general, for estimating a target value $p$ by an estimator $\hat{p}$ it is natural and usual to define the accuracy of the estimator by

$$
\begin{aligned}
\operatorname{Accuracy}(\hat{p}) & :=\epsilon(\hat{p}):=\sqrt{E(\hat{p}-p)^{2}} \\
& =\sqrt{\operatorname{Deviation}^{2}(\hat{p})+\operatorname{Bias}^{2}(\hat{p})} .
\end{aligned}
$$

Loosely speaking, for a second order kernel applied in (9) and any choice of $0<t^{*}<T$, the FRE has root-N $\left(\mathcal{O}\left(N^{-1 / 2}\right)\right)$ accuracy for dimension $d \leq 4$. For $d>4$ root-N accuracy is lost but then the FRE accuracy order is still the square of the FE/RE accuracy order (see Table 1). Moreover, it can be shown that root-N accuracy of (9) can also be achieved for $d>4$ by using higher order kernels in (9).

By definition (10) it is possible to relate the "expected" accuracy of the different density estimators to the number of simulated trajectories involved. However, simulating trajectories is not the only costly issue in the density estimation. For all estimators one has to evaluate a functional of the simulated trajectories. In case of the FE and RE estimators, this functional consists of a single summation, whereas for the FRE estimator a more complicated double summation needs to be evaluated. Therefore, for a proper comparison it is better to consider the complexity of the different estimators which is defined as the required computation cost for reaching a given accuracy $\varepsilon$. For instance, naive evaluation of the double sum in (9) would require a computational cost of order $\mathcal{O}(M N)$ in contrast to $\mathcal{O}(N)$ for the FE and RE estimators! Clearly, such an naive approach would have a dramatic impact on the complexity of the FRE. Fortunately, smarter procedures for evaluating this double sum exist, which utilize the small support of the kernel $K$. Particularly, in (Greengard and Strain 1991) it is shown for Gaussian kernels that this sum can be evaluated at a cost of $\mathcal{O}(N)$ in case $M=N$. In Table 1 we summarize the results of (Milstein et al. 2002) and list the accuracy and complexity of the FE, RE and the FRE where the latter is implemented with an efficient procedure for evaluating the double sum, for instance, according to the method of Greengard and Strain. For the FRE estimator we assumed $N=M$ and a second order kernel. It is because of the second order kernel that we have to distinguish for the FRE between $d<4$ and $d \geq 4$.

Remark. Naturally, all estimators FE, RE and FRE have a bias which involves a component due to application of the Euler scheme. Using results of (Bally and Talay 1996b) and (Bally and Talay 1996a), it is proven in (Milstein et al. 2002) that for all estimators the accuracy loss due to the Euler scheme applied with time discretization step $h$ is of order $\mathcal{O}(h)$ where, most importantly, the order coefficient may be chosen independent of the bandwidth $\delta$

\subsubsection{The choice of $t^{*}$ in the forward-reverse estimator}

The results concerning the order of accuracy and complexity of the FRE estimator in Sect. 2.2 do not depend on the particular choice of $t^{*}$ with $t<t^{*}<T$. However, the order coefficients do depend on this choice. To investigate this in more detail we consider Theorem 6.1. in (Milstein et al. 2002) for $d<4$. According to this theorem we have for $M=N$ and $\delta_{N}=N^{-1 / d} \log ^{1 / d} N$,

$E\left(\hat{p}_{F R E}-p\right)^{2}=\frac{D}{N}+o\left(N^{-1}\right)$

with

Table 1 Summary of accuracy and complexity of the forward (FE), reverse (RE), and forward-reverse (FRE) estimators

\begin{tabular}{lllll}
\hline Estimator & $\delta_{N}$ & Accuracy & Complexity & $\frac{C o m p l .\{F E, R E\}}{\text { Compl. }\{\text { FRE }}$ \\
\hline FE/RE & $N^{-1 /(4+d)}$ & $\mathcal{O}\left(N^{-2 /(4+d)}\right)$ & $\mathcal{O}\left(\epsilon^{-2-d / 2}\right)$ \\
FRE $d \leq 4$ & $N^{-1 / d} \log ^{1 / d} N$ & $\mathcal{O}\left(N^{-1 / 2}\right)$ & $\mathcal{O}\left(|\log \varepsilon| \epsilon^{-2}\right)$ & $|\log \varepsilon|^{-1} \varepsilon^{-d / 2}$ \\
FRE $d>4$ & $N^{-2 /(4+d)}$ & $\mathcal{O}\left(N^{-4 /(4+d)}\right)$ & $\mathcal{O}\left(|\log \varepsilon| \epsilon^{-1-d / 4}\right)$ & $|\log \varepsilon|^{-1} \varepsilon^{-1-d / 4}$ \\
\hline
\end{tabular}




$$
\begin{aligned}
D & :=\int r(u) \lambda^{2}(u) d u+\int r^{2}(u) \mu_{2}(u) q(u) d u-2 p^{2} \\
& =\int r(u) q^{2}(u) \mu^{2}(u) d u-p^{2}+\int r^{2}(u) \mu_{2}(u) q(u) d u-p^{2} \\
& =D_{(1)}+D_{(2)},
\end{aligned}
$$

where $r(u):=p\left(t, x, t^{*}, u\right)$ is the density of the random variable $X_{t, x}\left(t^{*}\right), q(u)$ denotes the density of $Y_{t^{*}, y}(T), \mu(u)$ is defined as the conditional mean of $\mathscr{Y}_{t^{*}, y}(T)$ given $Y_{t^{*}, y}(T)=u, \lambda(u):=q(u) \mu(u)$, and $\mu_{2}(u):=E\left(\mathscr{Y}_{t^{*}, y}^{2}(T) \mid\right.$ $\left.Y_{t^{*}, y}(T)=u\right)$. So we have in (11),

$$
\begin{aligned}
D_{(1)} & =\int r(u) q^{2}(u) \mu^{2}(u) \mathrm{d} u-\left(\int r(u) q(u) \mu(u) \mathrm{d} u\right)^{2} \\
& =\operatorname{Var}\left(q\left(X_{t, x}\left(t^{*}\right)\right) \mu\left(X_{t, x}\left(t^{*}\right)\right)=: f\left(t^{*}\right)\right.
\end{aligned}
$$

and

$$
\begin{aligned}
D_{(2)} & =\int r^{2}(u) E\left[\mathscr{Y}_{t^{*}, y}^{2}(T) \mid Y_{t^{*}, y}(T)=u\right] q(u) \mathrm{d} u-p^{2} \\
& =E\left[r^{2}\left(Y_{t^{*}, y}(T)\right) \mathscr{Y}_{t^{*}, y}^{2}(T)\right]-\left(E\left[r\left(Y_{t^{*}, y}(T)\right) \mathscr{Y}_{t^{*}, y}(T)\right]\right)^{2} \\
& =\operatorname{Var}\left(r\left(Y_{t^{*}, y}(T)\right) \mathscr{Y}_{t^{*}, y}(T)\right)=: g\left(t^{*}\right) .
\end{aligned}
$$

Since for $t^{*} \uparrow T, q \rightarrow \delta_{y}$ and for $t^{*} \downarrow t, r \rightarrow \delta_{x}$, it follows that

$\lim _{t^{*} \uparrow T} f\left(t^{*}\right)=+\infty$,

$\lim _{t^{*} \downarrow t} g\left(t^{*}\right)=+\infty$.

Further,

$\lim _{t^{*} \uparrow T} g\left(t^{*}\right)=\lim _{t^{*} \downarrow t} f\left(t^{*}\right)=0$.

Therefore, there exists an optimal choice $t_{o p t}^{*}$ which satisfies

$f\left(t_{\text {opt }}^{*}\right)+g\left(t_{\text {opt }}^{*}\right)=\min _{t<<}\left\{f\left(t^{*}\right)+g\left(t^{*}\right)\right\}$.

However, determination of such an exact optimum is not easy and also not necessary in practice. Experimantally, one should rather seek a $t^{*}$ such that both $f\left(t^{*}\right)$ and $g\left(t^{*}\right)$ are small. For instance, one could choose some candidates for $t^{*}$ and compare them by estimating the variances $f\left(t^{*}\right)$ and $g\left(t^{*}\right)$ roughly via classical (e.g. Parzen-Rozenblatt) approximations for $q$ and $r$ using small sample sizes. In Sect. 4.3 a heuristic method for the determination of $t^{*}$ will be presented. Without going into detail we note that similar considerations concerning a proper choice of $t^{*}$ apply for the case $d>4$.

\section{Test case: a stochastic BOD model}

The biochemical oxygen demand model presented in this section is often used in water quality determination of river and estuarine systems and is an extension of the so-called Streeter-Phelps model. In this model, the concentration levels of Carbonaceous Biochemical Oxygen Demand (CBOD), Dissolved Oxygen (DO), and Nitrogenous Biochemical Oxygen Demand (NBOD) are related to each other by a set of differential equations. With $b, o$, and $n$ representing the concentration levels $(\mathrm{mg} / \mathrm{l})$ of CBOD, DO, and NBOD, respectively, the system is given by

$$
\left[\begin{array}{l}
\mathrm{d} b / \mathrm{d} t \\
\mathrm{~d} o / \mathrm{d} t \\
\mathrm{~d} n / \mathrm{d} t
\end{array}\right]=\left[\begin{array}{ccc}
-k_{b} & 0 & 0 \\
-k_{c} & -k_{2} & -k_{n} \\
0 & 0 & -k_{n}
\end{array}\right]\left[\begin{array}{l}
b \\
o \\
n
\end{array}\right]+\left[\begin{array}{l}
s_{1} \\
s_{2}^{*} \\
s_{3}
\end{array}\right]
$$

where we defined $k_{b}:=k_{c}+k_{3}$ and $s_{2}^{*}:=k_{2} d_{s}+p_{o}-$ $r_{o}+s_{2}$. A description of the parameters used in the model, along with their units and typical values is given in Table 2. Although the concentrations are time dependent, the purpose behind the model is often to monitor the concentration levels within a fixed volume of water, flowing downstream a river. An underlying assumption is that the velocity of the water flow is constant and thus time and distance are linearly related. For more information about the model and its background, the reader is referred to e.g. (Stijnen 2002).

\subsection{Forward BOD model}

As for many environmental models, BOD is also subject to various uncertainties. These uncertainties can be incorporated into the model by adding white noise processes to each of the three equations of the model, effectively changing deterministic sources and sinks into stochastic ones:

$$
\begin{aligned}
{\left[\begin{array}{l}
\mathrm{d} B \\
\mathrm{~d} O \\
\mathrm{~d} N
\end{array}\right]=} & {\left[\begin{array}{ccc}
-k_{b} & 0 & 0 \\
-k_{c} & -k_{2} & -k_{n} \\
0 & 0 & -k_{n}
\end{array}\right]\left[\begin{array}{l}
B \\
O \\
N
\end{array}\right] \mathrm{d} t+\left[\begin{array}{l}
s_{1} \\
s_{2}^{*} \\
s_{3}
\end{array}\right] \mathrm{d} t } \\
& +\left[\begin{array}{ccc}
\sigma_{B} & 0 & 0 \\
0 & \sigma_{O} & 0 \\
0 & 0 & \sigma_{N}
\end{array}\right] \mathrm{d} W_{t},
\end{aligned}
$$

where the $\mathrm{d} W_{t}$ term denotes the Wiener process increment at time $t$. The stochastic BOD model is Markov and its variables Gaussian. Because the noise terms added do not depend on the values of $B, O$, or $N$, the model can be interpreted in both the Itô and Stratonovich sense. The stochastic equation (15) is linear, and an analytical solution is easily found.

A disadvantage of the simple additive white noise terms, is the possibility of getting negative concentration levels. This problem can be solved by scaling the noise term using a suitably chosen function, e.g.:

$$
s(x)= \begin{cases}0 & x<0 \\ \frac{1}{2}\left(\frac{2 x}{\tau}\right)^{\mathrm{d}} & 0 \leqslant x<\tau / 2 \\ 1-\frac{1}{2}(2(\tau-x) / z)^{\mathrm{d}} & \tau / 2 \leqslant x<\tau \\ 1 & \tau \leqslant x\end{cases}
$$


Table 2 Description and typical values for the parameters used in the BOD model

\begin{tabular}{llcc}
\hline Par. & Description & Unit & Value \\
\hline$k_{c}$ & Reaction rate coefficient & $(1 /$ day) & 0.763 \\
$k_{2}$ & Reaeration rate coefficient & $(1 /$ day) & 4.250 \\
$k_{3}$ & Sedimentation and adsorption loss rate for CBOD & $(1 /$ day) & 0.254 \\
$k_{n}$ & Decay rate of NBOD & $(1 /$ day) & 0.978 \\
$p_{o}$ & Photosynthesis of oxygen & $(1 /$ day) & 7.280 \\
$r_{o}$ & Respiration of oxygen & $(1 /$ day) & 7.750 \\
$d_{s}$ & Saturation concentration of oxygen & $(1 /$ day $)$ & 10.00 \\
$s_{1}$ & Independent source for CBOD & $(1 /$ day $)$ & 3.000 \\
$s_{2}$ & Independent source for OD & $(1 /$ day $)$ & 0.000 \\
$s_{3}$ & Independent source for NBOD & $(1 /$ day $)$ & 0.000 \\
\hline
\end{tabular}

with $d>0$ the order of the polynomial used, and $\tau$ the threshold below which the noise should be damped. Applying this to the BOD model would result in the following set of equations

$$
\begin{aligned}
{\left[\begin{array}{l}
\mathrm{d} B \\
\mathrm{~d} O \\
\mathrm{~d} N
\end{array}\right]=} & {\left[\begin{array}{ccc}
-k_{b} & 0 & 0 \\
-k_{c} & -k_{2} & -k_{n} \\
0 & 0 & -k_{n}
\end{array}\right]\left[\begin{array}{l}
B \\
O \\
N
\end{array}\right] \mathrm{d} t+\left[\begin{array}{l}
s_{1} \\
s_{2}^{*} \\
s_{3}
\end{array}\right] \mathrm{d} t } \\
& +\left[\begin{array}{ccc}
s(B) \sigma_{B} & 0 & 0 \\
0 & s(O) \sigma_{O} & 0 \\
0 & 0 & s(N) \sigma_{N}
\end{array}\right] \mathrm{d} W_{t},
\end{aligned}
$$

Although this solves the problem of negative concentrations, the linearity of the model is lost, along with the analytical solution and the Gaussian property. Being a test case for the forward-reverse estimator, the presence of an analytical solution of the presented model outweighs the disadvantage of a reduced practical meaning, as it allows for verification of the outcome. Therefore, it was decided to use the simple stochastic model (15) despite its obvious shortcoming.

Given this model, we want to determine $p(t, x, T, y)$, i.e. the probability density associated with the transition between a given start $(x)$ and end point $(y)$ indicated by two $(B, O, N)^{\mathrm{T}}$ vectors, at two distinct times $t \leq T$. Based on the forward model, it is possible to apply (3), which requires a set of $N$ realizations. These can be obtained from numerical integration of (15), as shown in Fig. 1a.

\subsection{Reverse BOD model}

In order to apply a forward-reverse estimator (Sect. 2.1.3), the reverse representation of the forward model needs to be derived. Since the presented BOD model is already given in the form prescribed by (1), straightforward substitution yields,

$a(t, X)=\left[\begin{array}{c}-k_{b} X^{(1)}+s_{1} \\ -k_{c} X^{(1)}-k_{2} X^{(2)}-k_{n} X^{(3)}+s_{2}^{*} \\ -k_{n} X^{(3)}+s_{3}\end{array}\right]$,

and
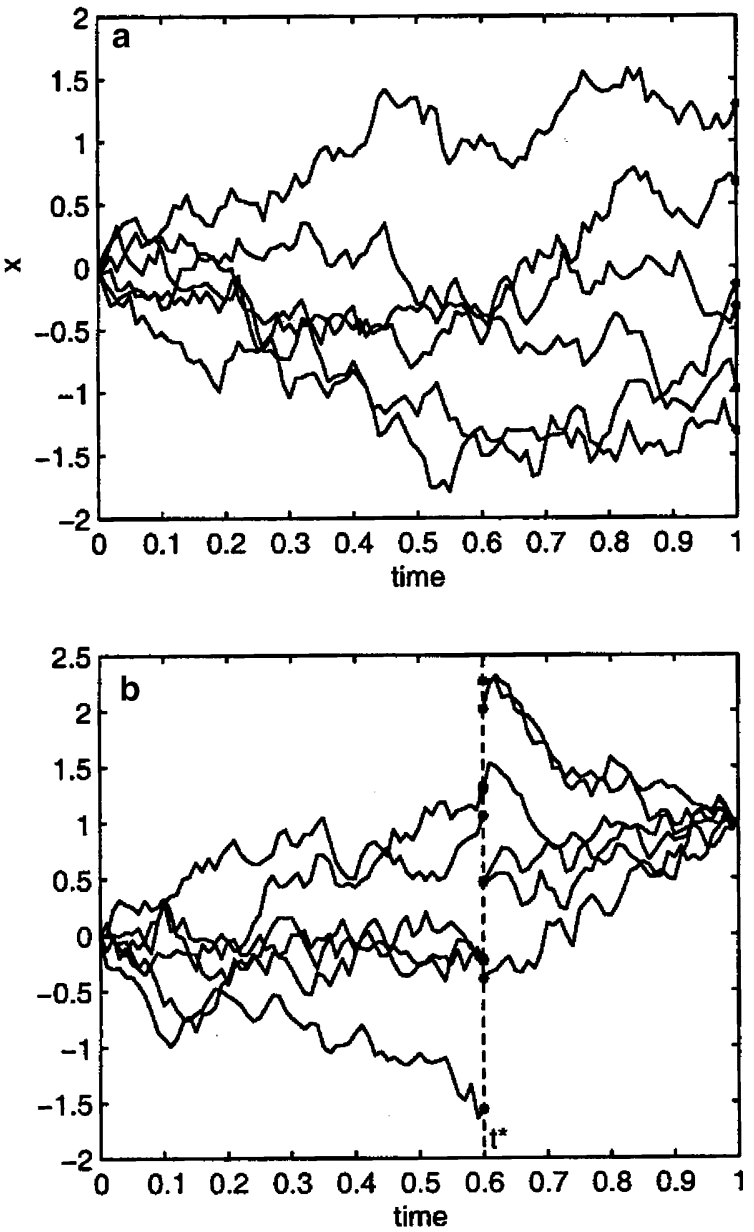

Fig. 1 Realization paths of a the forward process $\mathrm{X}$, and $\mathrm{b}$ the $\mathrm{X}$ and $\mathrm{Y}$ process used in the forward-reverse approach. The asterisk symbols indicate the end-points of the realizations that are used in the Monte Carlo estimator

$$
\begin{gathered}
\sigma(t, X)=\left[\begin{array}{ccc}
\sigma_{B} & 0 & 0 \\
0 & \sigma_{O} & 0 \\
0 & 0 & \sigma_{N}
\end{array}\right], \\
b=\sigma \sigma^{\mathrm{T}}=\left[\begin{array}{ccc}
\sigma_{B}^{2} & 0 & 0 \\
0 & \sigma_{O}^{2} & 0 \\
0 & 0 & \sigma_{N}^{2}
\end{array}\right] .
\end{gathered}
$$

Next, using Eqs. (5) and (6), the expressions for $\alpha$ and $c$ can be derived to be as follows: 


$$
\begin{aligned}
& \alpha(t, X)=\left[\begin{array}{c}
k_{b} X^{(1)}-s_{1} \\
k_{c} X^{(1)}+k_{2} X^{(2)}+k_{n} X^{(3)}-s_{2}^{*} \\
k_{n} X^{(3)}-s_{3}
\end{array}\right], \\
& c=k_{b}+k_{2}+k_{n} .
\end{aligned}
$$

with $X^{(1)}=B, X^{(2)}=O$, and $X^{(3)}=N$. For the reverse $\sigma$ process we have $\tilde{\sigma}=\sigma$. Now, with all variable terms in Eq. (4) known, the reverse process becomes

$$
\begin{aligned}
\mathrm{d} Y= & {\left[\begin{array}{c}
k_{b} X^{(1)}-s_{1} \\
k_{c} X^{(1)}+k_{2} X^{(2)}+k_{n} X^{(3)}-s_{2}^{*} \\
k_{n} X^{(3)}-s_{3}
\end{array}\right] \mathrm{d} s } \\
& +\left[\begin{array}{ccc}
\sigma_{B} & 0 & 0 \\
0 & \sigma_{O} & 0 \\
0 & 0 & \sigma_{N}
\end{array}\right] \mathrm{d} \tilde{W}(s)
\end{aligned}
$$

with initial condition

$Y\left(t_{0}\right)=y$,

and

$d \mathscr{Y}=\left(k_{b}+k_{2}+k_{n}\right) \mathscr{Y} d t$,

with

$\mathscr{Y}\left(t_{0}\right)=1$.

With all constants in the reverse model positive, both the $Y$ and $\mathscr{Y}$ processes are unstable, and grow exponentially. This relation is true in general; processes that are stable in the forward formulation will be unstable in the reverse formulation and vice versa. Just as for the forward formulation of the given BOD model, the reverse formulation is a linear system of equations, and the closed-form analytical solution is easily derived. How exactly the forward-reverse estimator is applied to obtain the probability density given earlier, is explained in the next section.

\section{Application of the forward-reverse estimator}

One way to carry out the forward-reverse estimation as presented in Sect. 2.1.3 is by means of a Monte Carlo simulation. Here, a number of realizations for both the forward and reverse models are generated (e.g. for the CBOD model this is done by numerically integrating Eq. (13) for the forward part and Eq. (14) and (16) for the reverse part). Based on the forward realizations, kernel estimation techniques are used to evaluate the transition density from the starting point of the simulation to the end point of each of the realizations of the reverse part, as illustrated by Fig. 1. A number of choices have to be made for the implementation of the algorithm. These will be discussed below.

\subsection{Numerical integration}

It is important to note that in the context of forwardreverse estimation, two sources of errors other than the kernel estimation exist. The first one is due to the use of a numerical scheme to approximate the SDE and the other originates from the use of Monte Carlo simulation. Numerous integration schemes can be used to generate numerical solutions of a stochastic differential equation. For our experiments, we used the Euler scheme, which was given by (2). The error of the numerical scheme can be reduced by using higher order schemes. The statistical error of the Monte Carlo simulation can be reduced by increasing the number of paths generated. Obviously, there should be a balance between these two sources of error. Reducing only one of the two will not always contribute to a reduction of the global error. As such, there should also be a balance between the stepsize used in the numerical scheme and the number of paths generated. This is discussed in more detail in (Schoenmakers and Heemink 1997).

\subsection{Kernel estimation}

The two most important aspects of kernel estimation are the kernel shape used, and the bandwidth parameter. These aspects are in close connection with the computational effort required to carry out a kernel estimation. This is of minor importance for single point evaluations, as used in forward density estimation, where a kernel estimation based on $N$ realizations can easily be implemented with $\mathcal{O}(N)$ time complexity. However, it is of major importance in cases where the approximated density function has to be evaluated at a large number of points. This is the case for forward-reverse density estimation. Naive algorithms easily lead to an $\mathcal{O}(N \cdot M)$ computational time, which is clearly prohibitively expensive. Greengard and Strain [4] developed an optimal algorithm for Gaussian kernels, based on the properties of Gaussian curves, that leads to an $\mathcal{O}(N+M)$ time complexity. One known drawback of their method, however, is the large constant multiplication factor. Unless the number of sample and evaluation points is very large, this constant will cause the computational time to be exceedingly long. We therefore decided to aim for a more modest $\mathcal{O}(M \log N)$ algorithm, with lower overhead, instead.

By using a parabolic kernel, the domain of influence of a realization is bounded, as opposed to the Gaussian kernel which has an unbounded support [3]. Because of this bounded support, most of the $N \cdot M$ kernel evaluations will be zero, which means that those realizations will not contribute to the probability density estimation at the given location. Based on this observation and the fact that our parabolic kernel only has a support radius of, say, $\phi$, it is possible to group the realizations in such a way that most of the zero kernel evaluations can be avoided, thus saving a lot of work. Given a set of $N$ forward realizations, $x_{i}$, in a $d$-dimensional space, with $1 \leq i \leq N$, we proceed as follows. 
1. Find the bounding box enclosing all realizations;

2. Create a regular grid such that the length of each side equals the kernel support, $\phi$;

3. Record which realizations are contained in which grid cell;

Once this information is known, the kernel estimation of a point at location $y$ can be obtained by following the procedure outlined below

1. Determine the cell containing $y$. Note that this cell does not necessarily have to be an existing cell, nor does it need to be inside the bounding box;

2. Find all cells that share at least one corner point with the aforementioned cell. This way, a total of $3^{d}$ cells are selected, including the cell containing $y$.

3. Retrieve all samples stored in the selected cells and use them to get a kernel estimation.

The distance from $y$ to a particle contained in any other cell is guaranteed to exceed the support range, $\phi$, because of the choice of cell size. Therefore, no information is lost, and the resulting kernel estimation is exactly the same as the one obtained using a 'brute-force' approach. In case of the FRE, the above three steps are simply repeated for each of the reverse realizations.

\subsubsection{Data structure}

Some remarks regarding the part of the algorithm where the data structure is set up are appropriate. This data structure stores the cells and the realizations contained in them. Once constructed, the data structure can be used to support fast evaluation of the kernel estimation based on these realizations. It is important to realize that only those cells containing realizations should be stored, which means a maximum of $N$ cells. Especially when the distributions of realizations is wide, compared to the kernel support, and the dimension of the problem is high, the potential number of cells that would otherwise be stored is enormous. To allow for both the selective storage and fast lookup of cells, a hashtable construction was used. Each entry in the table contains cells sharing the same hash value. The hash value is based on the coordinates of the cell which are carefully combined to allow for an even distribution of cells over table entries. Because the number of cells stored in each table entry is not known a priori and differs between entries, each entry merely contains a pointer to a linked list of cells. For similar reasons, each cell only contains a pointer to a linked list of the realizations it encloses, as shown in Fig. 2.

Retrieval of cells from the data structure proceeds in a series of steps. First, the hash value based on the cell's coordinates is computed. This value is then used to index the hashtable and access the list of all cells stored beginning at that link. Next, the list is traversed and the coordinates of each cell compared to the given coordinates, until either a match was found, or the end of the list reached. The realizations stored by the given cell can be retrieved likewise.

\subsubsection{Computational efficiency}

Although the algorithm described can be shown to have a worst-case $\mathcal{O}(N \cdot M)$ time complexity, it performed well in our experiments, showing the desired $\mathcal{O}(M \log N)$ behavior in most cases. In the cases where this time complexity was not achieved, the support size to realization distribution domain size ratio was found to be high. That is, either the support of the kernel was too large or the realizations were highly concentrated in a small region. Ultimately, it comes down to the problem that all realizations are contained in a small number of adjacent cells. When a kernel evaluation is made in nearby regions, most, if not all, realizations are included in the kernel evaluation leading to worst-case times. In the forward-reverse estimator, the optimal bandwidth used for kernel estimation depends on the number of forward realizations:

$\delta_{N}=N^{-1 / d} \log ^{1 / d} N$.

When the number of realizations is increased, the bandwidth and cellsize decrease and, as a result, the number of kernel evaluations made is reduced. Even for our three dimensional BOD model, $\mathcal{O}(M \log N)$ kernel estimations were made with $N$ well below 10,000.

\subsection{Combination point $t^{*}$ and number of tracks}

The remaining parameters to be discussed are the number of realizations generated and the location, $t^{*}$, where these realizations are combined using a kernel estimation. The effect the number of realizations have on the forward-reverse estimator is well known, at least, in terms of orders of convergence. Given a $d$-dimensional problem, the overall estimator accuracy is of order $\mathcal{O}\left(N^{-1 / 2}\right)$ for $d \leq 4$ and of order $\mathcal{O}\left(N^{-4 /(4+d)}\right)$, otherwise, with $N=M$ the number of forward and reverse realizations, respectively. Until now, the influence $t^{*}$ has on the estimator has not been thoroughly studied. In this section, we will show that in practice, this parameter is of utmost importance to the efficiency of the estimator. The experimental results given below are all based on the application of the forward-reverse estimator on the BOD model. Although many experiments were done to validate results, we only selected a single set of parameters to illustrate our results. The parameter settings are summarized in Table 3. The parabolic kernel was used for kernel evaluations, with a bandwidth of $(N / \log N)^{-1 / 3}$, which is equivalent to a support of $\sqrt{7 / 3}(N / \log N)^{-1 / 3}$.

\subsubsection{The influence of $t^{*}$}

In our first experiment, we determined the influence of $t^{*}$ on the estimator. The continuous parameter space of $t^{*}$ was first discretized, and only values of $t^{*}$ between 0.01 and 3.99, inclusive, at a regular interval of 0.01 were considered. For each of those values, 100 independent 
Fig. 2 Data structure used for storing realizations on which a kernel evaluation is based

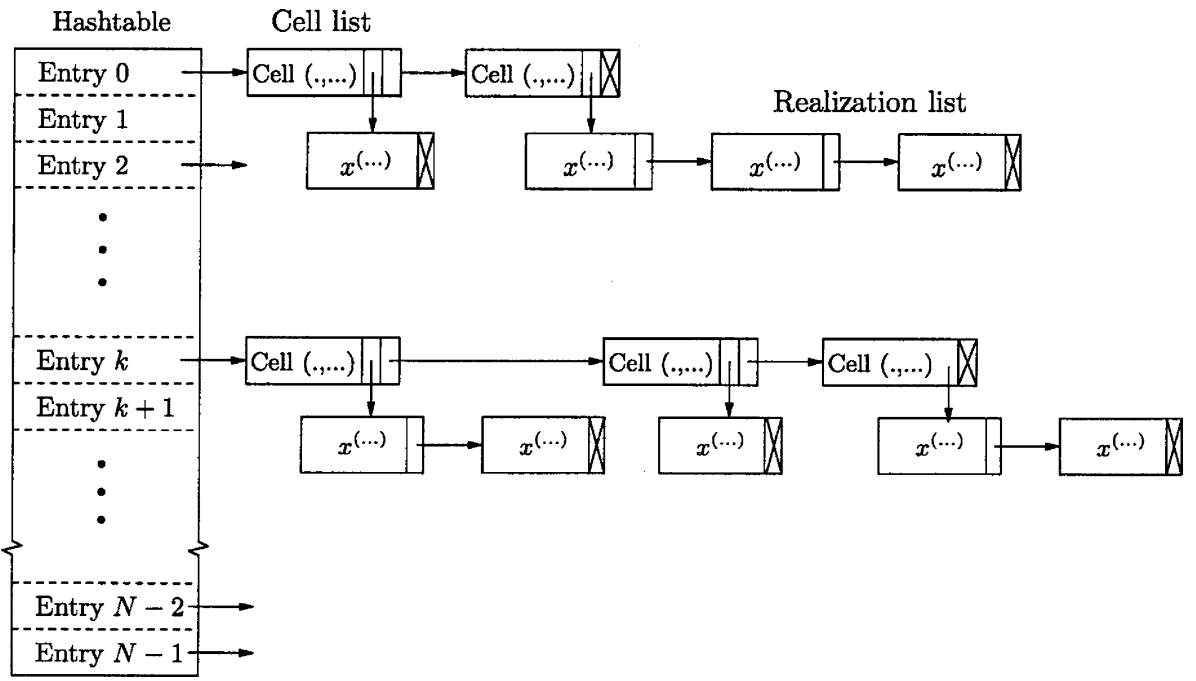

density estimations were done, to determine the mean and variance of the estimator. To avoid errors due to the use of a numerical integration scheme, the exact solution was sampled to provide realizations of the forward and reverse processes. Not only does this reduce the noise in the estimator, thus showing the influence of $t^{*}$ more clearly, it also greatly reduces the time requirement for the 39,900 estimations that were made. The results obtained for these runs, with $N=M=1,000$, are summarized in Fig. 3. For small $t^{*}$, the results show that many estimations are not very accurate, compared to the exact answer of approximately 0.13 . A closer look at the results for $t^{*} \in[2.7,4)$, given by Fig. 4 , however, shows that the FRE is indeed capable of giving accurate results for a range of $t^{*}$ values. The question remains what exactly causes the highly inaccurate answers for small $t^{*}$ ? The most obvious answer is to say that $N$ and $M$ are too small, and indeed, increasing them will result in more accurate estimations. In fact, all other reasons given below can be reduced to the lack of realizations in either direction. Nevertheless, they give valuable insight in the application of the FRE and therefore deserve mentioning.

Given the limited number of realizations used, one reason behind the erratic outcome of the FRE is the kernel estimation. During each density estimation the number of reverse realizations that fell within the kernel support was counted. The support domain was taken to be the smallest box enclosing all forward realizations, enlarged at both sides in each dimension by the band-

Table 3 Default parameter settings for the BOD model

\begin{tabular}{ll}
\hline Parameter & Value \\
\hline Start time $(t)$ & $t=0$ \\
End time $(T)$ & $T=4$ \\
Model parameters & $\sigma_{B}=1.5, \sigma_{O}=1.5, \sigma_{N}=1.0$, \\
& see further Table 2 \\
Initial point & $B_{t}=15, O_{t}=8.5, N_{t}=5$ \\
Default evaluation point & $B_{T}=3, O_{T}=9, N_{T}=0.1$ \\
Exact transition density & $0.13477720 \ldots$ \\
\hline
\end{tabular}

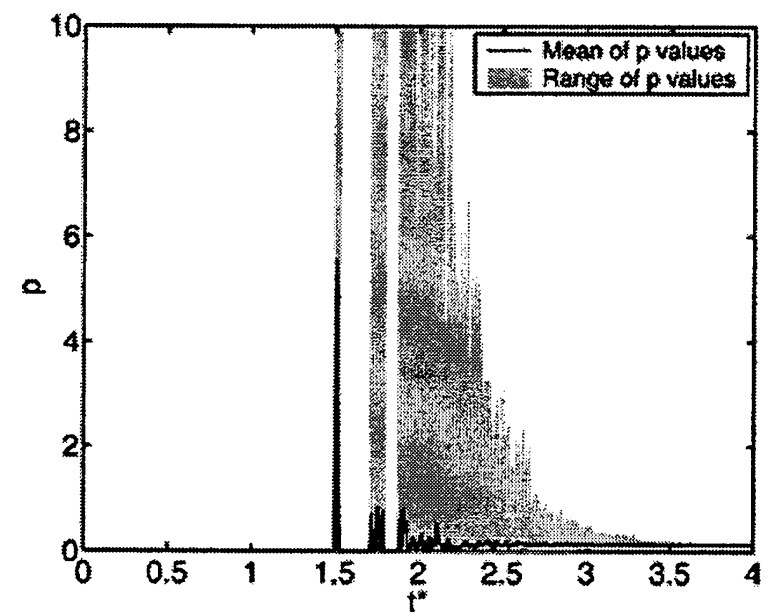

Fig. 3 Range and mean of $p$ values for different $t^{*} \in(0,4)$, based on 100 forward-reverse estimations at each of the $t^{*}$ locations, with $\mathrm{N}=\mathrm{M}=1,000$ and BOD parameters as given by Table 3

width of the kernel. This way, only those reverse realizations that are within the support can eventually attribute to the probability density. In Fig. 5 the average and range of these counts are plotted against $t^{*}$. For values of $t^{*}$ less than 2.5, the number of reverse realizations is zero in all but a few cases, causing the summation of all kernel estimations to evaluate to zero as well. In those cases where some kernel estimations were done, the density estimation is often exceedingly large. This is caused by a combination of factors. First, the process is unstable (see Fig. 14 for the covariance trace of the reverse process) which leads to an extremely flat Gaussian distribution. Combined with the small sample size, a realization that does end up inside the support domain can do so virtually anywhere. In addition, the already inaccurate estimation will then be inflated by the scaling factor $\mathscr{Y}$, which grows exponential in reverse time for the BOD model, resulting in a large overestimation of the probability density in the worst case. For the above problem, two solutions exist; (a) use more 


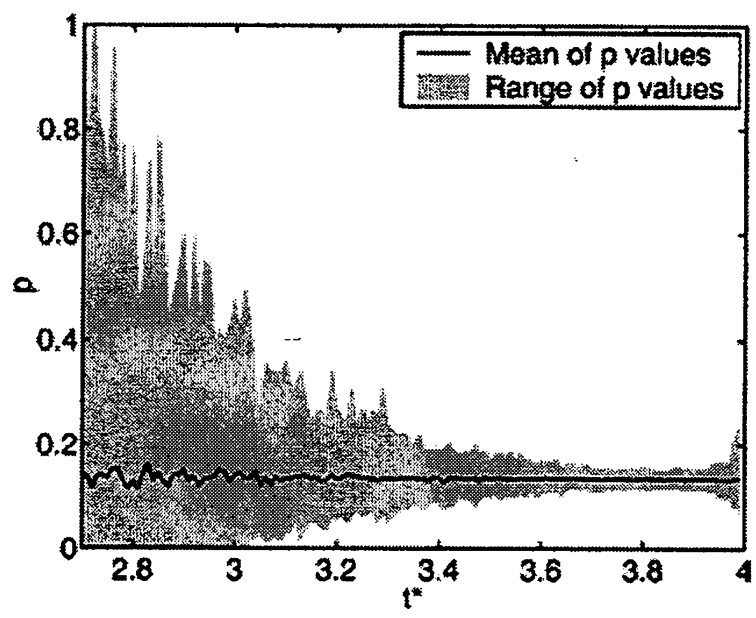

Fig. 4 Range and mean of $p$ values for different $t^{*} \in[2.7,4)$, based on 100 forward-reverse estimations at each of the $t^{*}$ locations, with $\mathrm{N}=\mathrm{M}=1,000$ and $\mathrm{BOD}$ parameters as given by Table 3

realizations to improve accuracy, and (b) avoid values of $t^{*}$ where the problem occurs. Both solutions will be considered below as we work towards a practical approach.

\subsubsection{The influence of the number of realizations}

The influence of the number of realizations on the estimation is perhaps best studied by looking at the variance in the estimation observed for different values of $N$ and $M$. Because of the results found earlier, we will henceforth only consider $t^{*} \in[2.8,4)$. Fig. 6 To start with, the ratio between variance obtained using $N=1,000$ and $N=10,000$, while keeping $M$ fixed at 1,000 was determined and is shown in Fig. 7. For $t^{*}<3.5$ there isn't any notable improvement with variance ratio's around 1. It is only for values of $t^{*}$ near $T$ that considerable improvements are made. The reason for the lack of

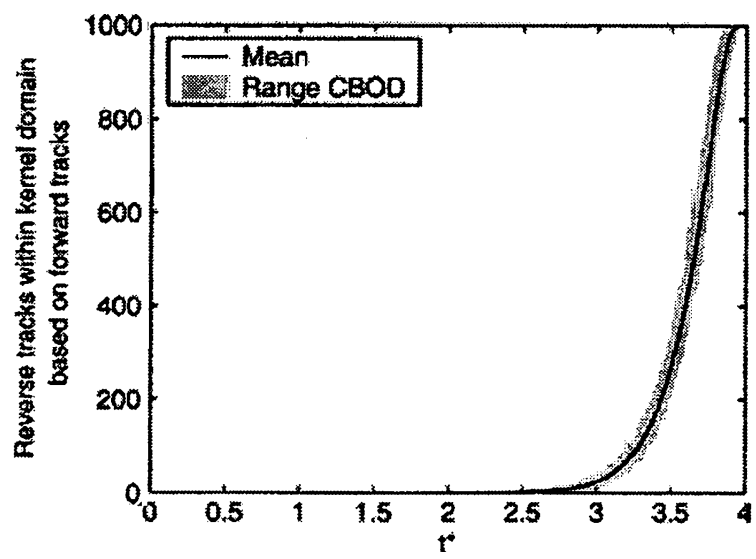

Fig. 5 Range and mean of number of reverse realizations that fall within the support of the kernel estimation at different $t^{*} \in(0,4)$. Based on 100 forward-reverse estimations with $\mathrm{N}=\mathrm{M}=1,000$ and BOD parameters as given by Table 3

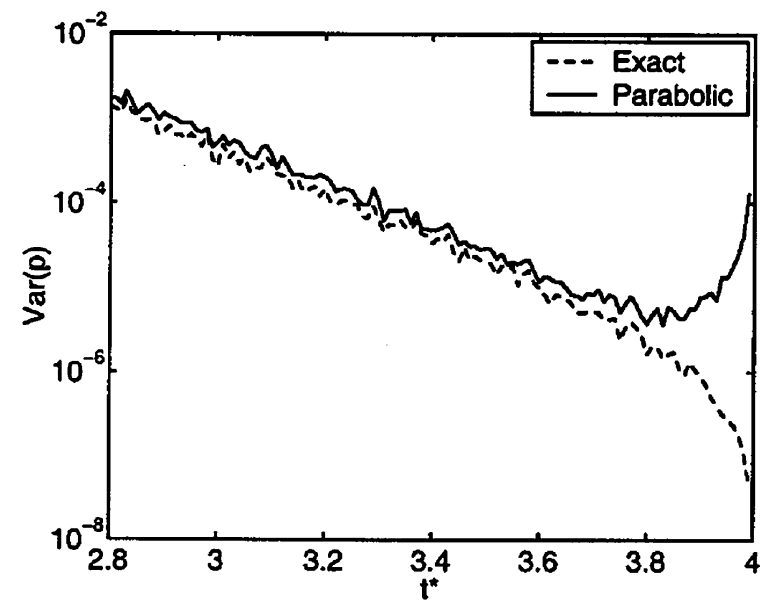

Fig. $65 \mathrm{~cm}$ Variance in $p$ values for different $t^{*} \in[2.8,4)$, based on 100 forward-reverse estimations at each of the $t^{*}$ locations, with $\mathrm{N}=\mathrm{M}=10,000$ and BOD parameters as given by Table 3 , using parabolic kernel estimation (solid line) or the analytical transition density function (dashed line)

improvement is the fast rate at which the reverse process diffuses, and the reduction of non-zero kernel evaluations that follows. Therefore, improving the kernel estimation accuracy will only result in marginal improvements.

In the reverse experiment, we increase $M$ from 1,000 to 10,000 while maintaining $N=1,000$ and again determine the variance improvement. The results are shown in Fig. 8. This time, there is no improvement in the estimate for $t^{*}$ near $T$, with a ratio of 1 . Better results are obtained by moving $t^{*}$ towards the intitial point $t$ where the ratio increases to the maximum possible value of 10 . In between, the kernel estimation's accuracy and reverse Monte Carlo accuracy are clearly not balanced, or more precisely, their balance was disturbed by the increase of

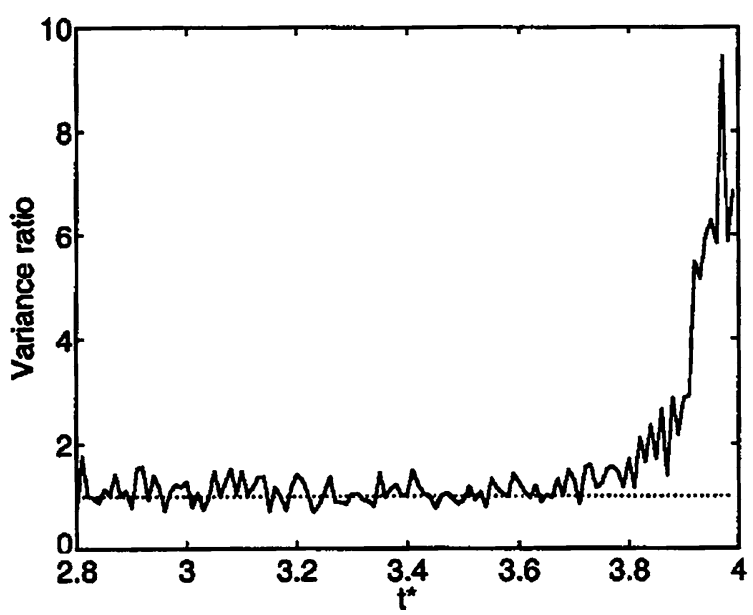

Fig. 7 The effect of increasing the number of forward realizations $\mathrm{N}$ from 1,000 to 10,000 , while retaining $\mathrm{M}=1,000$. Measured in the ratio of variance in $p$ variables for different $t^{*} \in(2.8,4]$, before and after the change. Based on 100 forward-reverse estimations with BOD parameters as given by Table 3 


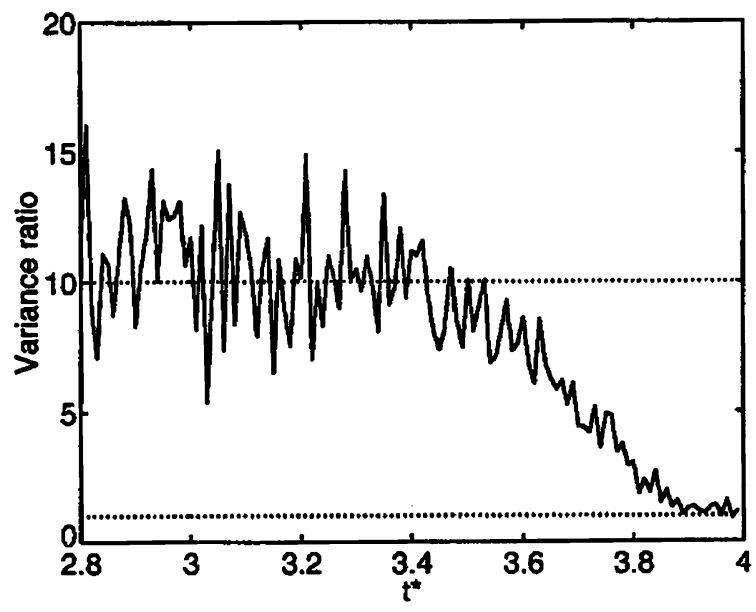

Fig. 8 The effect of increasing the number of reverse realizations $M$ from 1,000 to 10,000 , while retaining $\mathrm{N}=1,000$. Measured in the ratio of variance in $p$ variables for different $t^{*} \in(2.8,4]$, before and after the change. Based on 100 forward-reverse estimations with BOD parameters as given by Table 3

reverse realizations. In case both $M$ and $N$ are simultaneously increased to 10,000 , the results are as given by Fig. 9. Here, the results are good overall with variance improvement ratio's of around 10 .

\subsubsection{The choice of the number of realizations and $t^{*}$}

Increasing the number of realizations adds to the computation time of the estimator. Therefore, we would like to know the best combination of $N, M$, and $t^{*}$ to reach a certain level of accuracy in the estimation. Going back to the results obtained with $N=M=1,000$, we will explain how this can be done. In Fig. 10 the variance in 100 estimations is plotted against $t^{*}$ along

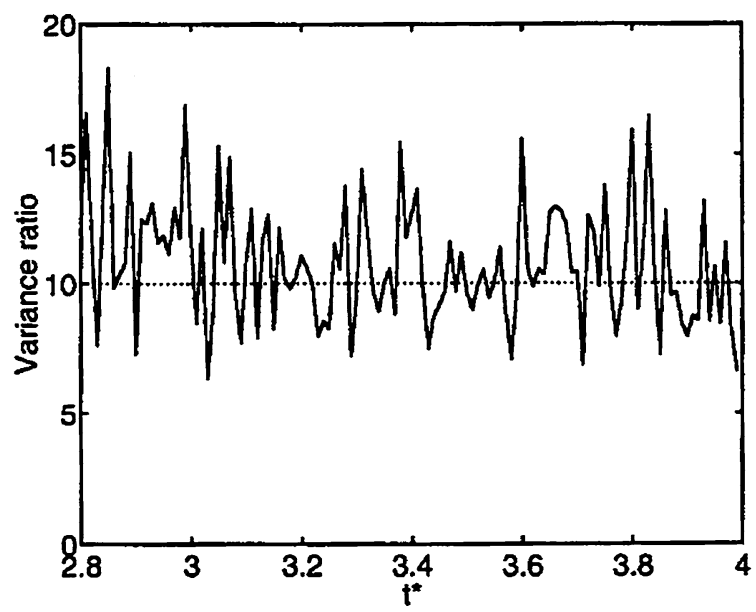

Fig. 9 The effect of simultaneously increasing the number of forward and reverse realizations from 1,000 to 10,000. Measured in the ratio of variance in $p$ variables for different $t^{*} \in(2.8,4]$, before and after the change. Based on 100 forward-reverse estimations with BOD parameters as given by Table 3

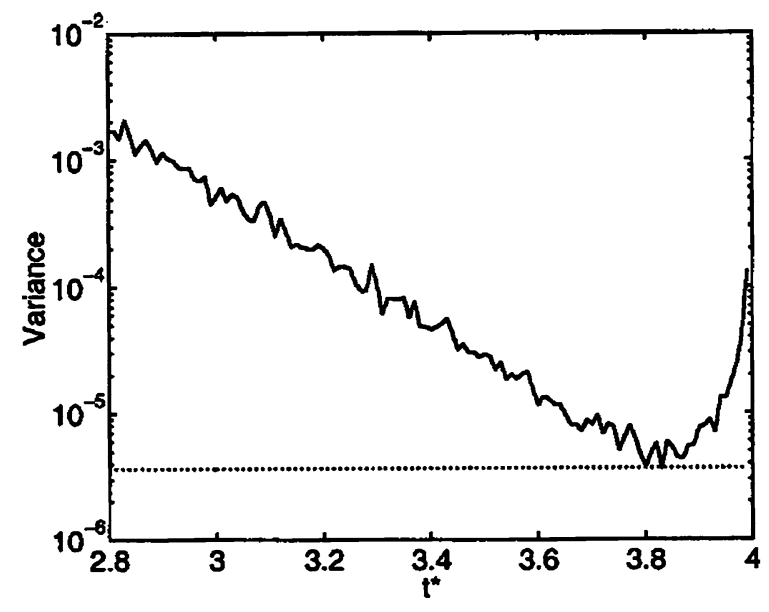

Fig. 10 Variance in $p$ values for different $t^{*} \in[2.8,4)$, based on 100 forward-reverse estimations at each of the $t^{*}$ locations, with $\mathrm{N}=\mathrm{M}=10,000$, parabolic kernel estimation, and BOD parameters as given by Table 3

with a dotted line indicating the minimum level of variance. Given the number of realizations, this is clearly the best result we can obtain, but at what expense? Because of the sampling, we could only measure the kernel estimation times for each run. The average runtime in seconds for each $t^{*}$ is given by Fig. 11. It can be seen that the kernel time at the assumed optimal $t^{*}$ is high, compared to those for lower valued combination points. This raises the question whether the given $t^{*}$ is indeed optimal, or if lower variations could be reached using a different $t^{*}$ and more realizations, at the same computational time. Suppose it were possible to decrease variance linear with the number of forward and reverse realizations (we assume $N=M$ from now, unless specified otherwise) infinitely, and that such increase will affect the computation time linearly. The fact that this is practically impossible,

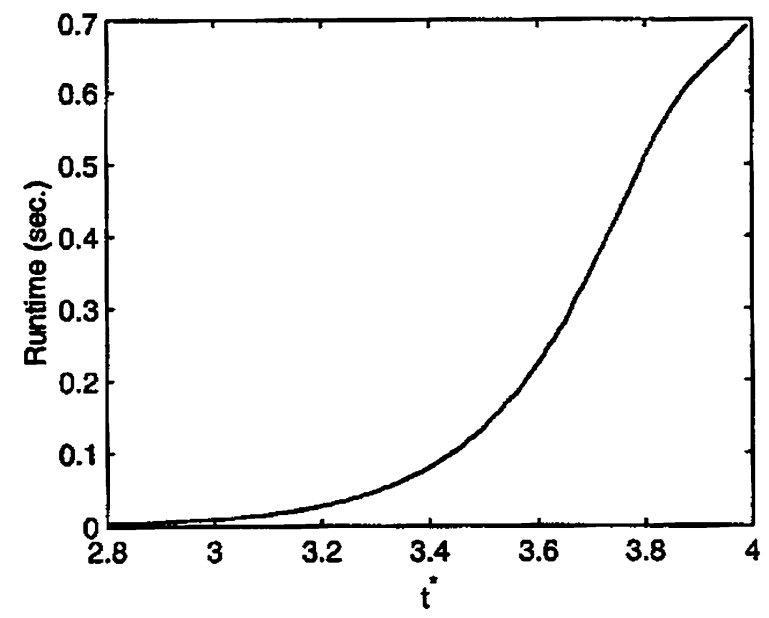

Fig. 11 Average kernel estimation times recorded for different $t^{*}$ $\in[2.8,4)$. Based on 100 forward-reverse estimations with $\mathrm{N}=\mathrm{M}=10,000$ and $\mathrm{BOD}$ parameters as given by Table 3 


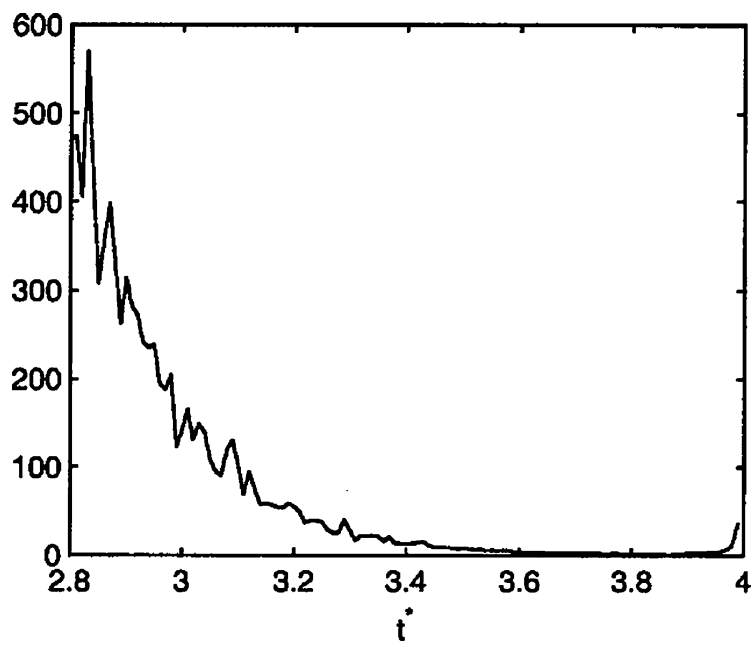

Fig. 12 Factor by which number of forward and reverse tracks should at least be multiplied in order to obtain a density estimation with a variance equal to the one indicated by the dotted line in Figure 10

with the kernel evaluation order and the need to decrease the numerical integration stepsize, will only strengthen our reasoning. Then, based on the minimum variance seen in Fig. 10, it is possible to determine the factor by which the number of realizations be multiplied to achieve the same level of variance, as shown in Fig. 12. By multiplying the kernel times with this factor, we obtain optimistic computation time for kernel evaluation to get iso-variance estimations Fig. 13. The location where this time is minimum, say $t^{*}$ Low, is shifted towards $t$ slightly from the location of the original minimum variance, $t^{*}{ }_{\text {High }}$. Under the assumption that evaluation of the numerical integration scheme for the forward and reverse is equally

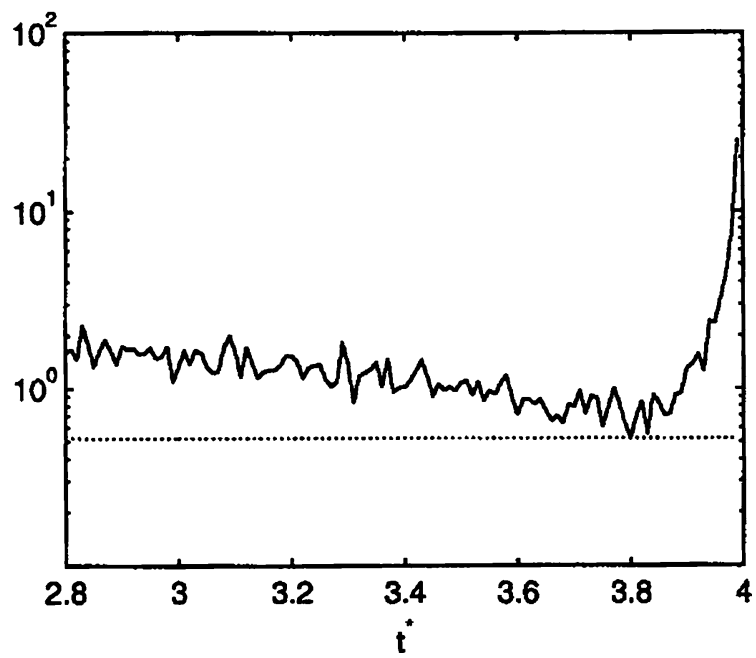

Fig. 13 Idealistic time required to complete kernel estimation in case the number of realizations is chosen such that the variance in density estimation is the same for all $t^{*} \in[2.84$ )

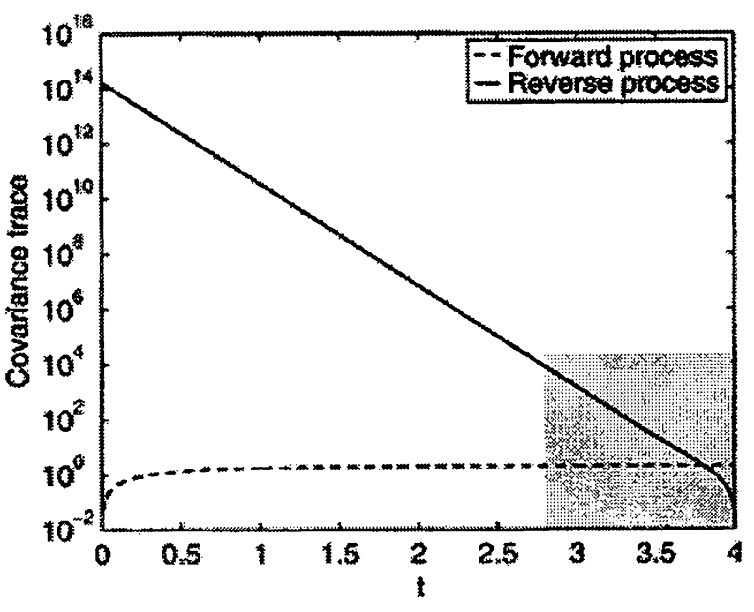

Fig. 14 Trace of the covariance matrices of the forward and reverse processes, plotted against time. Time $t=4$ is the end time of the simulation and hence the start time of the reverse process

expensive in terms of computational cost, the real optimum, $t^{*} \mathrm{Opt}$, is limited as follows:

$t_{\text {Low }}^{*}<t_{\text {Opt }}^{*} \leq t_{\mathrm{High}}^{*}$.

The lower bound will shift further towards $t_{\text {High }}^{*}$ when numerical integration is included and the kernel evaluation times are determined more realistically. In case the reverse process is much more expensive per timestep than the forward process, the lower bound may shift a little towards $t$.

Normally, it is not practically feasible to use the above approach to determine a near-optimal $t^{*}$, because of the computational effort required, at least, not in a similar level of detail. As suggested earlier the process can be done roughly but that will result in an crude selection of $t^{*}$. It would be better if, somehow, we could determine this location directly from the two processes. For the BOD process, and other Gaussian processes in general, it was noted that the difference in variance (for higher dimensional systems, the covariance matrix' trace) between the forward and reverse processes was reflected in the variance of the estimate, as can be seen by comparing Figs. 14 and 10. This would lead to a simple and elegant solution to the problem of finding a good value for $t^{*}$. Simply generate a small set of realizations and repeatedly increase them by use of the numerical integration scheme. While doing so, maintain the sum of the variance of each component of the realizations, for each timestep. Next, find $t^{*}$ where the absolute difference between the obtained forward and reverse variances is at its minimum and use this location as the combination point for the full-run. The results from experiments based on a simple diffusion algorithm (see [10] for further information) where the analytical variance is known, were very promising as the values found for $t^{*}$ proved to be good indications of the optimal location. 


\section{Performance evaluation of FRE versus FE}

In this section, the FRE and FE are compared to each other both in terms of order of convergence of the error in the estimation, and in terms of accuracy to computing time.

\subsection{The order of convergence}

The order of convergence of the accuracy of the estimated probability density can be approximated by running a number of estimations for each of a set of values for $N$. The standard deviation and bias of the estimation are then determined for each $N$, and the results plotted on a log-log scaled figure. From this figure, the order of convergence corresponds directly to the slope of the plotted line and can hence be easily determined. We did a large number of experiments with different choices for $t^{*}$ and location of the evaluation point $y$, in $p(t, x, T, y)$. Because of the similarity of the results, we only show the results for the BOD model with parameters as given by Table 3. For the FRE, we used $N=M=2^{i}$, with $i=5, \ldots, 15$, and $t^{*}=3.803800$, while for the FE, $n=2^{j}$, with $j=5, \ldots, 18$ was used. In both estimators, the transition density was estimated 10,000 times to get accurate values for the standard deviation and bias. In addition, the numerical integration was replaced by sampling the known distributions. Figs. 15 and 16 respectively show the standard deviation and squared bias in the forward and forward-reverse estimations, along with dotted lines indicating the theoretical orders of convergence, plotted to the number of realizations.

The results match the theoretical results very closely, with the exception of the squared bias for the forwardreverse estimator which shows a convergence of $\mathcal{O}\left(N^{-1.1}\right)$, faster than the $\mathcal{O}\left(N^{-1}\right)$ plotted. It is known(Milstein et al.2002) that the squared bias for problems with $d<4$, is $o\left(N^{-1}\right)$. Therefore, the results

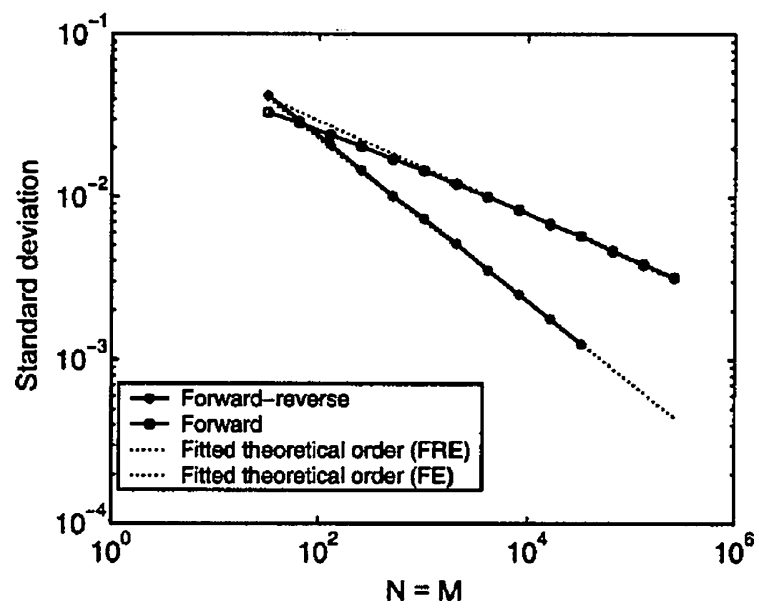

Fig. 15 Convergence of the standard deviation in the estimated transition density

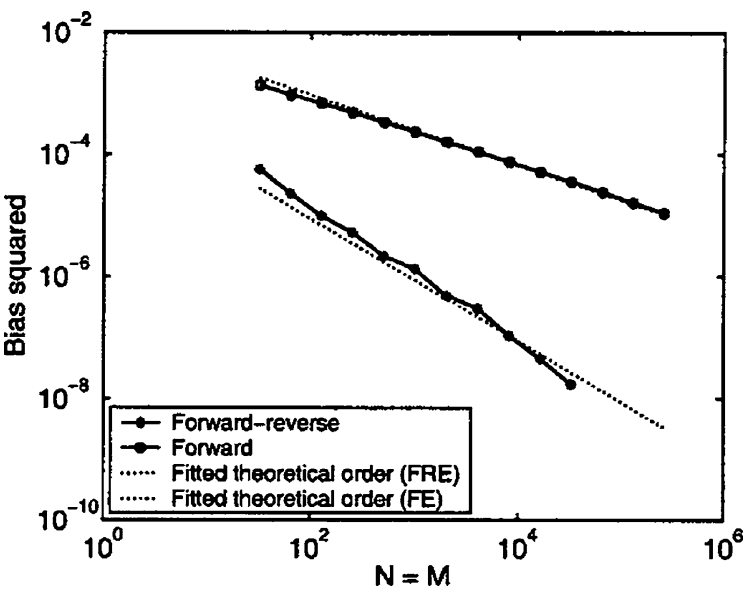

Fig. 16 Convergence of squared bias in the estimated transition density

still match the theory, although the convergence rate found may be overestimated because of the relatively small number of realizations used.

\subsection{Computational costs}

For a realistic comparison between the two estimators, the numerical integration scheme has to be taken into account since it accounts for a major part of the total computational cost. For example, the run-time of the forward estimation can be expressed as follows

runtime $_{F E}=\tau_{\text {integration step }} \frac{N \cdot(T-t)}{\Delta}+\tau_{\text {kernel eval }} \cdot N$

where the constants $\tau_{\text {integration step }}$ and $\tau_{\text {kernel eval }}$ represent the execution time of a numerical integration step and a kernel evaluation respectively, and $\Delta$ is the timestep used in the integration scheme. The constants will differ slightly for each implementation and computer platform, but are easily measured. The value for $\tau_{\text {integration step was }}$ found to be several times larger than $\tau_{\text {kernel eval }}$, mostly because of the cost of generating random numbers. As a result, even at a moderate timespan $(T-t)$ and large $\Delta$, most of the computation time will be spent on generating realizations.

The run-time for the forward-reverse estimator is more difficult to express because it is not linear in the number of realizations used. Especially the kernel estimation time is hard to predict because it depends on the forward and reverse distributions at the combination point, which in turn depend on the $x$ and $y$ in the density function. Despite this, we still want to compare the FE and FRE in terms of computation time. This can be done by recording the simulation time of a forward-reverse estimator. This time can then be substituted in (20) to determine the matching number of realizations in the FE simulation. After running an equivalent FE simulation, the results of the two estimators can be compared. 
Table 4 Number of realizations that can be used in the FE to obtain a run time similar to the FRE, when using $N=M=$ 262,144 , and a stepsize as indicated

\begin{tabular}{llll}
\hline Stepsize & 0.1 & 0.01 & 0.001 \\
\hline$N$ & 574,950 & 293,389 & 266,052 \\
\hline
\end{tabular}

The above approach was applied to the BOD model with parameters as given by Table 3 . For numerical integration, the Euler scheme as given by (2) was used with stepsize, $\Delta$, of $0.1,0.01$ and 0.001 . The number of realizations used in the FRE was $N=M=2^{i}$, with $i=7, \ldots, 18$. The value of $t^{*}$ was related to the stepsize, resulting in value of $3.8,3.80$, and 3.804 , respectively. As an example, Table 4 gives the equivalent number of realizations to be used in the $\mathrm{FE}$, in case $i=18$, or similarly, $N=M=262,144$;

The results of the experiments are given by Figs. 17 and 18. Curves with smaller timesteps will have larger associated run-times and will hence start more to the right in the plot. Both the standard deviation and squared bias of the FRE are consistently smaller than those of the FE, while obtained at a similar computational effort. Therefore we can conclude that the FRE is indeed superior to FE, even for small numbers of realizations.

\section{Conclusions}

We have applied the recently introduced forward-reverse (Milstein et al.2002) to a stochastic environmental BOD model. After the derivation of the forward-reverse representation of the model, we investigated a number of issues that required special attention in order to make application of the forward-reverse method efficient in a practical sense. We argued that the calculation of the

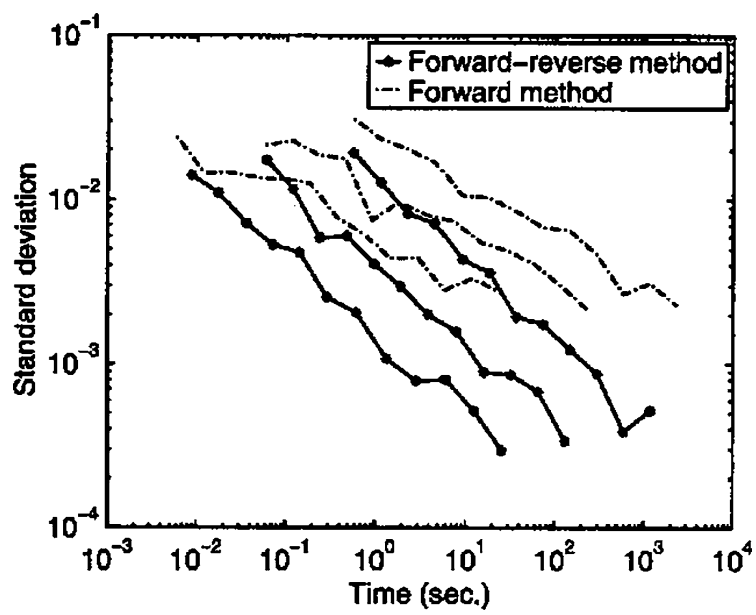

Fig. 17 Standard deviation in transition density estimation for the forward and forward-reverse estimators, plotted against simulation time. From left to right $\Delta=1 / 10,1 / 100$ and $1 / 1000$

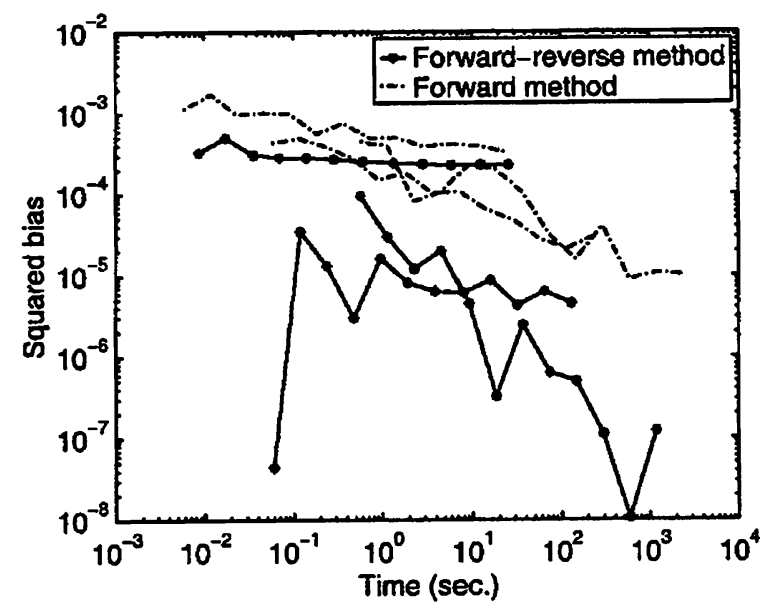

Fig. $185 \mathrm{~cm}$ Squared bias in transition density estimation for the forward and forward-reverse estimators, plotted against simulation time. From left to right $\Delta=1 / 10,1 / 100$ and $1 / 1000$

kernel estimator must be implemented with care and presented an $\mathcal{O}(M \log N)$ scheme. In Sect. 4.3, the relation between combination point $t^{*}$ and the computational efficiency has been analyzed. We presented an efficient practical approach for determining the appropriate value for $t^{*}$. It was then shown that the forwardreverse estimator indeed achieves an $\mathcal{O}\left(N^{-1 / 2}\right)$ accuracy when applied to the BOD model, compared to an $\mathcal{O}\left(N^{-2 / 7}\right)$ accuracy for the forward estimator (see Table 1). The comparison of the estimation error to the run-time clearly shows the advantage of using the forward-reverse estimator. Especially for problems where a high accuracy is required, the FRE will be substantially faster than the FE, as seen in Fig. 17. These results are encouraging and show the practical use of the forwardreverse approach for density estimation in environmental risk analyses.

Acknowledgements The authors would like to thank G.N. Milstein for helpful discussions and comments.

\section{References}

Bally V, Talay D (1996a) The law of the Eulerscheme for stochastic differential equations I: convergence rate of the density. Monte Carlo Methods Appl 2:93-128

Bally V,Talay D (1996b) The law of the Euler scheme for stochastic differential equations I: convergence rate of the distribution function. Probab Theory Relat Fields 104:43-60

Devroye L, Györfi L (1985) Nonparametric density estimation: the $\mathrm{L}_{1}$. View Wiley

Devroye L (1986) Non-uniform random number generation. Springer, Berlin Heidelberg New York

Greengrad L, Strain J (1991) The fast Gauss transform. SIAM J sic stat Comp 12:79-94

Hammersley JM, Handscomb DC (1964) Monte Carlo methods. Wiley, London

Jazwinsky AW (1970) Stochastic processes and filtering theory. Academic, New York

Kloeden PE, Platen E (1992) Numerical solution of stochastic differential equations. Springer, Berlin Heidelberg New York 
Loucks DP, Stedinger JR, Haith DA (1981) Water resources systems planning and analysis. Prentice-Hall, New York

Milstein GN (1995) Numerical integration of stochastic differential equations. Kluwer, Dordrecht

Milstein GN, Schoenmakers JGM (2002)Monte Carlo construction of hedging strategies against multi-asset European claims stochastics. Stochastics Rep 1-2:125-157

Milstein GN, Schoenmakers JGM, Spokoiny V (2002) Transition density estimation for stochastic differential equations via forward reverse representations. Bernoulli (submitted)

Schoenmakers JGM, Heemink AW (1997) Fast valuation of financial derivatives. J Comput Finance 1(1):47-67
Schoenmakers JGM, Heemink AW, Ponnambalam K, Kloeden PE (2002) Variance reduction for Monte Carlo simulation of stochastic environmental models. Appl Math Model 26: 785-795

Silverman BW (1986) Density estimation for statistics and data analysis. Chapman and Hall Ltd, New York

Stijnen JW (2002) Numerical methods for stochastic environmental models Delft University of Technology. Ph.D. Thesis, Defalt University of Technology

Thomson DJ (1987) Criteria for the selection of stochastic models of particle trajectories in turbulent flows. J Fluid Mech 180:529-556 